\title{
POST TONSILLECTOMY LATE ONSET BLEED WITH DENGUE FEVER BEING THE CAUSE
}

\author{
MAJOR (Dr) ABHIJITH. Y. V ${ }^{1}$, Dr. RAMESH. $M^{\mathbf{2}}$ \& Dr. SURAJ ${ }^{\mathbf{3}}$ \\ ${ }^{I}$ Assistant professor, Department of Paediatrics BGS Global institute of Medical Sciences (BGSGIMS\&H), \\ BGS Health and Education, Bengaluru, Karnataka, India \\ ${ }^{2}$ Professor and Unit chief, Department of Paediatrics, (BGSGIMS\&H), Karnataka, India \\ ${ }^{3}$ Internee, Department of Paediatrics,(BGSGIMS\&H), Karnataka, India
}

\begin{abstract}
The late onset post tonsillectomy bleed is the least common variety of the three types described. It usually occurs beyond 10 days of tonsillectomy surgery, due to secondary causes not related directly to the surgery. We report one such rare case of a 10 year old school going male child, who presented to the hospital as very sick looking patient, 09 days after discharge with medical history of fever, sore throat, and cough with hematemesis, abdominal pain, vomiting and loose stools. Examination findings were hyperpyrexia and visible petechiae, but washemodynamically stable and systemic findings showed abdomen to be tense, other systemic examinations were normal. An early diagnosis of the cause (Dengue fever), for the bleed in this patient was very crucial in the successful management of the case, even with its rarity of occurence.

KEYWORDS:Post tonsillectomy status, Late onset bleed, Thrombocytopenia\&Haemorrhage
\end{abstract}

Received: Mar 31, 2020; Accepted:Apr 22, 2020; Published:Jun 01, 2020; PaperId.: IJMPSJUN20207

\section{INTRODUCTION}

Among the paediatric age group, Dengue fever has been increasing in prevalence over the past decades, making India a dengue endemic. There islimited availability of data through online reporting for occurrence of both dengue and post tonsillectomystatus, as a causefor haemorrhages occurring at the same time. For any of the Otolaryngologist practicing in dengue prone area, it is necessary to consider that there is a possibility that patients may face this and necessary prevention should be done to avoid the unnecessary morbidity and mortality ${ }^{1}$.

The Aedesegypti mosquito is the cause for the dengue fever. It belongs to the flavivirusfamily, which is a day time biting mosquito. Dengue is an endemic in Asia, the Pacific, Africa and the America.The Aedesegypti breeds in stagnant waters such as collection of water in broken bottles or water based air coolers., ${ }^{2,3}$

The transmission is from a mosquito to the human, primarily. The vertical transmission of the dengue virus (DENV) is low as per the evidences (from pregnant mother to her baby), mostly due to the infection during the pregnancy, due to which the babies may suffer from prematurity, distress at birth, low birthweight ${ }^{\mathbf{4 , 5}}$.

There are 4 serotypes of Dengue fever causing virus (DENV). Interestingly, all of them present with nearly the same clinical picture. At post infection, the immunity is lifelong only for the particular type of serotype. Whereas, the immunity for rest of the serotypes last only for few months. Hence, when the individuals who are already immune to one of the types of serotype, and he gets infected with a new serotype, can develop the Severe form of Dengue, and manifest with worse prognosis. There is damage to the endothelium by the cytokine released due to increased T-cell activation when the cells expressing Fc receptor antibody are taken in by the virus, when 
infected by a new serotype virus. This causes the capillaries to leak and also disseminated intravascular coagulation.

Initially,the simple variety of Dengue fever was seen in infants younger than 2 years. The severe form of the dengue fever was seen in children between the age groups of 2-15years. Off late, the clinical manifestation scenario has changed. The severe form of it is seen in children less than 2 years also. The same is observed frequently in the age groups of 16-45 years, which are seen to have severetarget organ damage due to above mentioned capillary leak and DIC.

\section{INCUBATION PERIOD}

The multiplication of the virus begins in the midgut, and then disseminated to the salivary glands after feeding on the person infected with dengue virus. The terminology extrinsic incubation period (EIP), is very important. This is the time taken to infect a new host from the time of ingestion of the virus by the mosquito. Generally, it is about 8-12 days in the temperatures that are ambient $\left(25-28^{\circ} \mathrm{C}\right)$. The daily temperature fluctuations, initial viral concentration and the genotype of the virus are some of the other factors responsible for the alterations in the time the mosquito takes for virus transmission. Interesting fact is that, once infected, the mosquito can transmit the virus for its life time.

\section{DENGUE CHARACTERISTICS}

The characteristics are like a flu type. It seldom causes death, affects the infants, young children and adults. The duration of the symptoms can be from two days to about a week, after the infected mosquito has bitten in the last 4-10 days. It has been classified under 2 categories by the world health organization:-

- Dengue without warning signs

- Dengue with warning signs

- Severe Dengue

The suspicion for this type of fever should arise, when the temperature is very high $\left(40^{\circ} \mathrm{C} / 104^{\circ} \mathrm{F}\right)$ and is also having any of these symptoms like: joint aches, muscle aches, severe form of headache, vomiting, nausea, rashes.The critical phase of dengue fever is usually seen in the severe form of it, which lasts for a period of 3 to 7 days after the onset of the illness. At this point of time, the patient slowly becomes afebrile, and the manifestation of severe dengue fever with warning signs can happen.

This is a very dangerous complication, which can also be a cause for the fatality of the patient, more so due to the pathophysiological mechanisms like the severe bleeding manifestations, plasma leakage due to the endothelial damage, respiratory distress. The Warning signs that the treating physician has to be vigilant for are as follows- bleeding from the gums/mucosa, easy fatigability, persistence of the vomiting, vomitus with blood, fast breathing and restlessness.

The dengue fever pattern is a continuous or saddle back appearance on charting body temperature, on the day four of illness the peak comes down.Later on, can have the recurrence of fever for next 3-4 days. The manifestation of the rashes is usually in the initial first two days and the pattern is macular to maculopapular to morbilliform blanching type rashes on the third to fifth day in the trunk region. They spread centrifugally and spare the soles and the palmar aspect. The stage of convalescenceis slow, which may be associated with arthralgia, fatigue for longer period and some amount of depression. The complications include the severe organ damage. 


\section{INVESTIGATIONS}

After the first few days of the infection, the virus can be isolated by various methods like the RT-PCR (reverse transcriptase-polymerase chain reaction). This method is sensitive generally, but the drawback is the need of the specialized equipment. In the developing nations like ours, it is a great difficulty to make it available at all places. The training of the staff for these sophisticated methods is also a big hurdle.

The other method of testing the presence of the virus with easy availability is the NS1 antigen test, a virusproduced protein. It is a rapid diagnostic tool, wherein the result is available within a span of 20 minutes. The added advantage in a country like ours and the other developing nations is the non-requirement of sophisticated equipment availabilityor qualified technician.

The enzyme-linked immune-sorbent assays (ELISA) test is a type of serological test method. This test is able to depict the time period of the infection, like recent or past infection (analysis based on the presence of IgM and IgG antidengue antibodies). This method detects $\operatorname{IgM}$ antibodies within a period of 07 days from the time of infection and highly detectable after 14 days to 28 days from the onset of the illness. These antibodies can be seen detected in the tests for a period of about 90 days, are indicative of a recent infection from the DENV virus. The other type of antibody (IgG antibody) takes more time than $\operatorname{IgM}$ in the formation. However, they are indicative of past infection and remain in the body for years together.

\section{TREATMENT}

Dengue fever treatment is not a specific type, but there are standard guidelines laid out for the ease of the management of the patient. They are symptomatically managed as per the WHO guidelines. The main stay of treatment in the severe form of dengue feveris mainly the management of total fluid volume. The management of complications is also equally important at every stage of the disease, once they set in.

On the other hand, various surgeries are done on a day to day basis with very good success rates. One of the most commonly conducted surgeries by the Otorhinolaryngologists is the tonsillectomy. Children usually undergo tonsillectomy due to some recurrent infections or tonsillar hyperplasia. There are various grades of tonsillitis, to classify it and decide upon for surgery based on the clinical setting and the history pattern.

These children can come with a large variety of symptoms from obstructive sleep apnoea, hyperactivity and behavioural problems to recurrent infective symptoms such as lymphadenopathy, fever, halitosis, pain etc.But, over the past several years, sleep disordered breathing is the leading cause rather than recurrent infections. This is one of the important causes in children, who undergo tonsillectomy for disturbed sleep. Also, they are the ones who are more prone for post-operative haemorrhage and need for post-operative readmission. The bleeding rates in this type of surgery are only $1-4 \%$. It is seen that, most of it is occurring in the first two weeks after the surgery ${ }^{6}$.

One such procedure related interesting case report is discussed below. However, the cause of the bleed in this patient is a rare entity, which makes the presentation interesting and the efforts for continued knowledge sharing using the modern methods of communication to fellow colleagues. 


\section{CASE REPORT}

A ten year old school going child, only born issue to non-consanguineous marriage, hailing from Kengeri town, Bengaluru city, Karnataka state; underwent elective adeno-tonsillectomy in a tertiary care hospital with unremarkable peri- operative period. However, the child was re-admitted with a suspicion of sepsis, 09 days afterthe procedure and discharge from the hospital; now presenting with the complaints of fever, sore throat, and cough with hematemesis, abdominal pain, vomiting and loose stools.

On examination the child looked sick with hyperpyrexia and visible petechiae, but was hemodynamically stable and systemic findings showed abdomen to be tense. However other system examinations were normal.

The initial investigations of complete blood count (CBC) revealed platelet counts of $44 \mathrm{X} 10^{3} / \mu \mathrm{l}$ with lymphocytic predominance and total count of 5 X 103/ $\mu 1$, Renal and liver function tests were within normal limits. Blood and sputum culture reports showed no growth. The Ultrasonography (USG) of abdomen and pelvic region showed hepatomegaly with moderate ascites, right-sided minimal pleural effusion, and pericholecystic fluid with gall bladder wall thickening.However the serial CBC done based on the clinical setting,revealed adepletion in platelet count going as low up to $28.9 \times 10^{3} / \mu 1$. Dengue serology test using ELISA NS1 antigen came reactive with a value of $134.7(<9:$ nonreactive). The right-sided pleural effusion (minimal) was appreciated in the radiological test, the chest x-ray.

The child was admitted in Paediatric intensive care unit (PICU) and was managed conservatively, who responded well. Hewas discharged with the final diagnosis of post tonsillectomy late onset bleed with dengue fever being the cause.

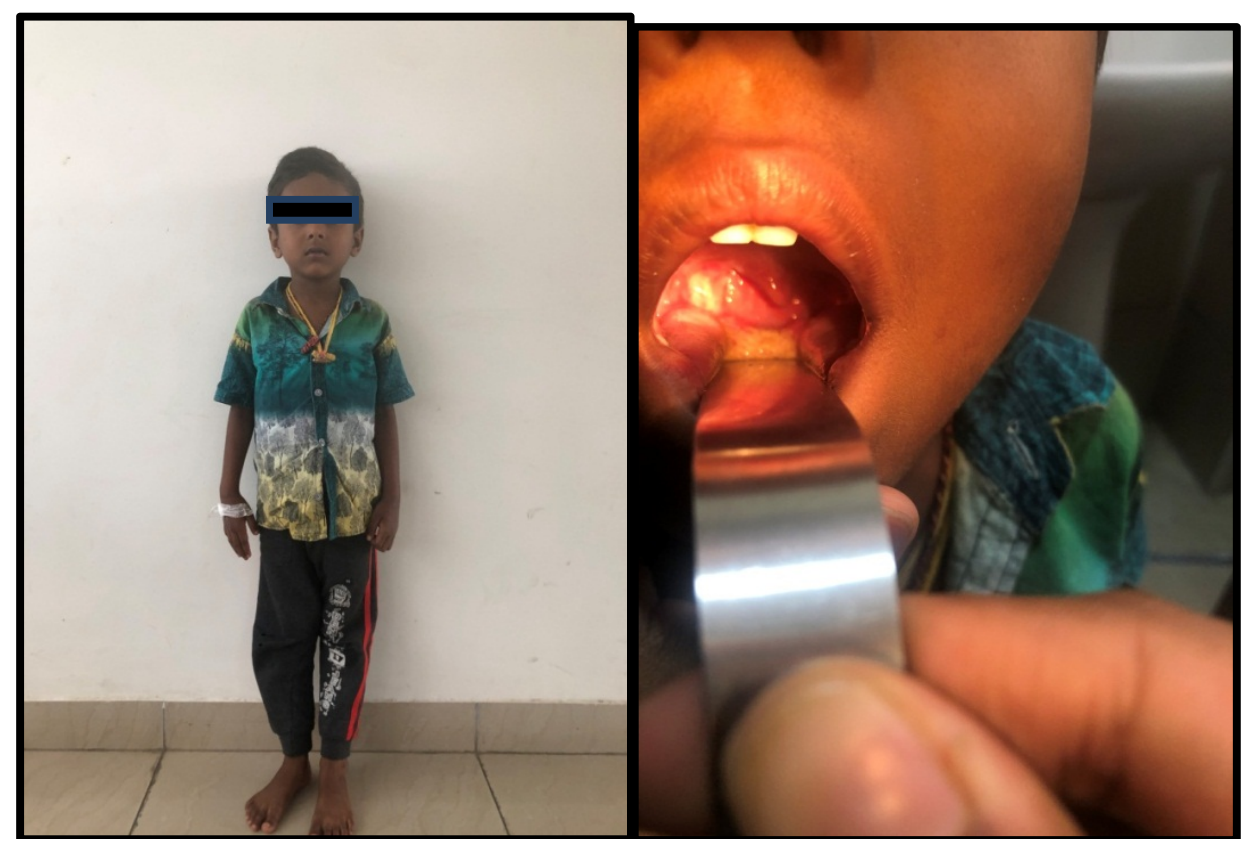

Figure 1

\section{CONCLUSIONS}

Post tonsillectomy bleed is categorized into three types: a) Early/primary bleeding which occurs during the first 24 hours after operation, is considered to be directly related to the surgical technique; b) Delayed/secondary bleeding which occurs on days 1- 10, usually due to sloughed off Escher (most commonly on post-operative days 2-7); c)Late bleeding which occurs beyond 10 days (least common), considered to be due to secondary factors not directly related to tonsillectomy, as 
seen in this case.

Dengue on the other hand, is aninfection that is viral in origin and is caused bythe strains belonging to family flaviviridae. It is endemic and occurs year-round in India, including urban and suburban areas, with a death rate of $4.2 \%{ }^{6}$. Haemorrhage and/or pulmonary congestion from fluid overload on convalesant or critical phase remains to be common cause of mortality.

\section{REFERENCES}

1. Josephine Grace C, Rojo, MD. www.aseanorl2019.org; Concurrence of late post tonsillectomy bleed and dengue fever in a 17 year old female; first reported case; Philippines.

2. Medlock, J.M., et al., Analysis of the potential for survival and seasonal activity of Aedesalbopictus (Diptera: Culicidae) in the United Kingdom. Journal of Vector Ecology, 2006. 31(2): p. 292-304.

3. Ketaren, S. Otniel, et al. "Environmental health aspect in health emergency management (a case study: SinabungVulcanous Eruption)." Int. J. Appl. Nat. Sci. 5 (2016): 47-56.

4. Romi, R., F. Severini, and L. Toma, Cold acclimation and overwintering of female Aedesalbopictus in Roma. Journal of the American Mosquito Control Association, 2006. 22(1): p. 149-151.

5. Dhevarajan, S., et al. "SPR_ SODE Model for Dengue Fever." International Journal of applied mathematical and statistical sciences", ISSN (2013): 2319-3972.

6. Pouliot, S.H., et al., Maternal Dengue and Pregnancy Outcomes A Systematic Review. Obstetrical \& Gynecological Survey, 2010. 65(2): p. 107-118.

7. Basurko, C., et al., Maternal and foetal consequences of dengue fever during pregnancy. European Journal of Obstetrics \& Gynecology and Reproductive Biology, 2009. 147(1): p. 29-32.

8. MomoLokko, Christiana Naa, and Francis Lokko. "Clinical Presentation and Management of Human Ebola Virus Disease in Sub-Saharan Africa." International Journal of Medicine and Pharmaceutical Science (IJMPS) ISSN (P) (2016): 2250-0049.

9. WindfurJPI, SchloendorffG, BaburiD, Kremer B. serious post tonsillectomy haemorrhage with and without lethal outcome in children and adolescents. Int J PediatirOtorhinolaryngol, 2008 Jul; 72(7); 1029-40. PMID: 18455808

10. DHEVARAJAN, S., et al. "ASYMPTOTIC STABILITY OF SPR_SODE MODEL FOR DENGUE." IMPACT: International Journal of Research in Applied, Natural and Social Sciences (IMPACT: IJRANSS) 1. 6, Nov 2013, 51-56

11. SrinivasraoMuthenni, Andrew P Morse, Cyril Caminade and Suryanarayana Murthy Upadhyayula. Dengue burden in India. Emerging Microbes and Infection, 2017 Aug 9. PMID:28790459

12. J. Johnson, $H$ McLaren, $M$ mahadevan and $R G$ Douglas "clinical characteristics of obstructive sleep apnoea versus infectious adenotonsillar hyperplasia in children" International journal of PediatricOtorhinolaryngoscopy, vol, 116,pp 177180,2019 . 

